\title{
Analysis of Quercetin and Its 3-Glycosides by Paper Partition Chromatography
}

Sir :

Owing to the resemblance of the chemical structure, solubility, and spectrum, it is difficult to separate and determine quercetrin, isoquercitrin (quercetin-3-glucoside prepared from leaves of mulberry), quercitrin (quercetin-3-rhamnoside), and rutin (quercetin-3-rhamno-glucoside from buckwheat).

But we have obtained good results in the analysis of these flavonoid pigments by paper partition chromatography.

The method employed is as follows :

1) In the one-dimensional descending chromatography, strips of filter paper "Toyo No.4" $(2 \times 40 \mathrm{~cm}$. $)$ were used.

2) The best developing solvent was the water-rich layer of the three component system, consisting of 50 parts ethyl acetate, 2 parts glacial acetic acid, and 50 parts distilled water.

3) The saturated aqueous solution of basic lead acetate was used as the colouring reagent and the spots were observed under ordinary and ultraviolet lights.

The Rf values of these flavonoid pigments obtained in this experiment were as follows : quercetin 0.80 , quercitrin 0.67 , isoquercitrin 0.43 , and rutin 0.10 .

When yellow precipitates obtained by decomposition of these glycosides with dilute $\mathrm{H}_{2} \mathrm{SO}_{4}$ were chromatographed, the spot of quercetin, the aglycone of these glycosides, was detected.

'The solution obtained by filtering off the yellow precipitates was neutralized and concentrated in vacuo, and the sugars in this concentrate were chromatographically analysed. We detected glucose from isoquercitrin, rhamnose from quercitrin and both of these sugars from rutin on chromatograms by PARTRIDGE's method [Nature, 164, 443, (1949)].

An alcoholic solution of rutin was incompletely decomposed by dilnte $\mathrm{H}_{2} \mathrm{SO}_{4}$ and neutralized. By the chromatographic analysis of this solution, isoquercitrin was detected. From this fact, it was proved that the disaccharide combined at 3-position of rutin was rhamno-glucoside.

These flavonoid pigments extracted from 10 to 20 spots of chromatograms were identified with original flavonoids by spectral analysis.

It was an interesting fact that the Rf values of these glycosides were parallel to those of the corresponding sugars.

\begin{tabular}{l|c||l|c}
\multicolumn{1}{l|}{ Flavonoids } & Rf value & Sugars & Rf value \\
\cline { 1 - 3 } & 0.10 & Disaccharide Lactose & 0.10 \\
Rutin & 0.43 & Glucose & 0.12 \\
Isoquercitrin & 0.67 & Rhamnose & 0.21 \\
Quercitrin & & 0.39 \\
\hline
\end{tabular}

In other words, solubilities of these flavonoid pigments depend on the nature of sugars combined at 3-position.

Biochemical Laboratory,

Faculty of Agricuiture, Kyushu University, Fukuoka 